\title{
Relay Selection and Power Allocation with QoS Support for Secondary Users in Cognitive Radio Networks
}

\author{
Yang Yu \\ Wuhan No.2 Ship Development Design Institute, Wuhan, China \\ 544738692@qq.com
}

\begin{abstract}
Keywords: power allocation, relay selection, cognitive radio networks, quality-of-service (QoS).
Abstract. In this paper, we study the problem of joint relay selection and power allocation in a cognitive radio (CR) network. Different from conventional algorithms that only aim at maximizing source node utility, we also take into account the utility of the SU which acts as the relay. On the premise of meeting the quality of service (QoS) requirements of the source and the relay, our goal is to maximize the system throughput subject to primary user (PU) received interference power constraints and total transmit power constraints on the source and the relay. In the context, two scenarios where the relay node involves relay diversity and dual-hop transmission are considered respectively. Furthermore, simulation results and comparisons are presented to illustrate the performance of the proposed schemes.
\end{abstract}

\section{Introduction}

Emerging wireless services envisage the challenge that the available spectrum is becoming increasingly scarce. Within the current spectrum regulatory framework, however, a lot of licensed spectrum bands are underutilized most of time. Cognitive radio (CR) [1], which can intelligently identify unused licensed spectrum bands and then adaptively use them, is proposed as the key technology towards efficient spectrum usage. Meanwhile, cooperative technique is regarded widely as an effective means for improving the rate and range of communication in various types of wireless networks, including CR networks.

The benefits provided by the usage of cooperative technique in CR networks for spectrum sharing were investigated in [2], [3]. Generally speaking, besides link reliability, cooperative transmission can also improve system throughput through not only spatial diversity but also spectrum diversity [3]. Aiming at these benefits, various schemes have been proposed. Among them, relay selection and power allocation schemes, which affect the performance of cooperative CR networks significantly, have recently received great attention [4]-[6]. However, most of the existing works focus on maximizing the capacity [4]-[6] or improving the end-to-end reliability [4] of the source node based on the assumption that the relay does not intend to transmission its own information and devotes all its power to help the source transmit. However, this assumption may be not reasonable for practical CR networks. Compared to traditional wireless communication systems, the available spectrum resource is time-varying and geographically unbalanced [3] within CR systems. Therefore, it is expensive and inefficient to deploy complex network infrastructure like dedicated relay, and it is preferred that SUs implement information relaying. In the case that a SU acts as the relay node, not only the performance of the source but also the performance of this SU's own data transmission should be optimized. When the relay's own data transmission is considered, the source and the relay in a three-node cooperative CR network will operate in multiple spectrum bands simultaneously [3], [7]. This will make us face more complex relay selection and power allocation problems.

In this paper, we investigate joint relay selection and power allocation for AF relaying in a three-node CR network, which consists of a source, a relay, and a secondary access point (AP). The relay is selected from a set of SUs, and the source and all the relay candidates regard the AP as their common destination. The interference introduced to the PU resulting from the secondary transmission is kept below a specified threshold. Aiming at maximizing the overall utility of the CR network while supporting QoS for the source and the relay with different minimum rate requirements, two scenarios 
where the relay node implements relay diversity and dual-hop transmission are considered. In the proposed optimal algorithms, we determine the most profitable relay by completely searching all available relay candidates. For a given relay selection, the power allocation in the first scenario can be formulated as a convex optimization problem and thus a closed-form solution can be derived easily based on dual method. However, as to the scenario where the relay node involves dual-hop transmission, the resulting power allocation problem is non-convex, and hence hard to solve in general. To tackle this, we introduce a power proportionality factor at the relay to decompose the non-convex optimization into two levels. Based on dual method and search method at each level respectively, the optimum can be achieved.

\section{System model}

As shown in Fig.1, we focus on a centralized cooperative CR network where the transmission of the secondary source (denoted by s) is assisted by $\mathrm{SU} i, i=1,2, \ldots, L$, which is selected from a potential relay set. Both the source and the relay intend to send their own data to a AP (denoted by d). In this paper, we employ the AF cooperation protocol. Adopting discontiguous-OFDM [3] as the physical-layer technique, both the source node and the relay node are able to transmit multiple packets on multiple bands simultaneously using single-radio equipment.

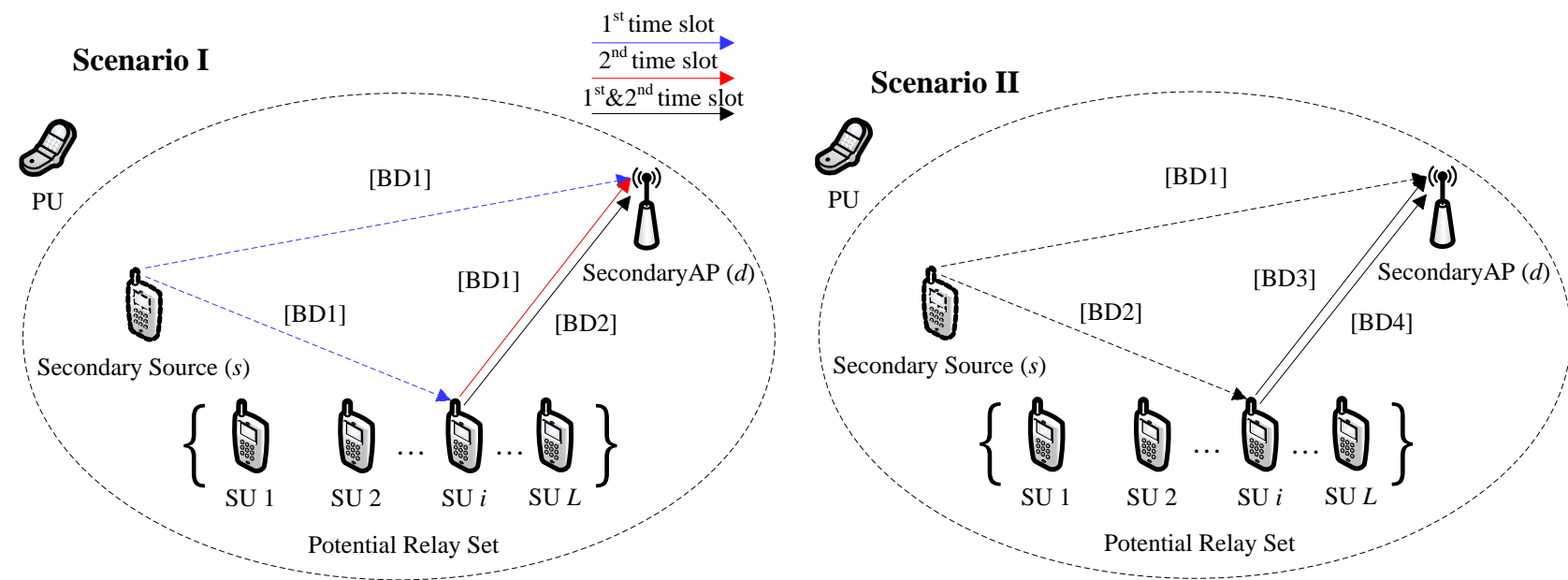

Fig. 1 The structure of the cooperative CR network.

In the context, channels used for helping the source relay information can be divided into two categories: a relay channel and a dual-hop channel [7]. Correspondingly, the cooperative relay transmission happens in two scenarios, i.e., Scenario I where the relay helps the source transmit with a relay channel, and Scenario II where the relay helps the source transmit with a dual-hop channel as shown in Fig.1.

\section{Transmit Power Constraints and System Throughput for Scenario I}

In Scenario I, relay diversity transmission happens and an AF cooperative transmission procedure occurs in two time slots. Suppose all channel coefficients are fixed during the time of interest.

We assume that two orthogonal bands, band 1 (BD1) and BD2, are used in the three-node network as illustrated in Fig. 1. The relay diversity transmission happens on BD1. Denote $p_{s, j}[t]$ and $p_{i, j}[t]$ as the transmit power of the source and the relay for $\mathrm{BD} j, j=1,2$, in time slot $t, t=1,2$. Then their power allocation vectors can be expressed as

$\mathbf{p}_{\mathrm{s}}=\left[p_{s, 1}[1], p_{s, 2}[1], p_{s, 1}[2], p_{s, 2}[2]\right]$, and $\mathbf{p}_{\mathbf{i}}=\left[p_{i, 1}[1], p_{i, 2}[1], p_{i, 1}[2], p_{i, 2}[2]\right]$, where $p_{s, 2}[1]=p_{i, 1}[1]=p_{s, 1}[2]=$ $p_{s, 2}[2]=0$.

In the first time slot, the source broadcasts its own information using BD1. In the second time slot, the relay amplifies the signal received from the source and relays it to the AP using BD1. Denote $g_{s i}$, $g_{s d}$ and $g_{i d}$ as the channel coefficients from the source to the relay, the source to the destination and the relay to the destination, respectively. Assume the additive white Gaussian noises (AWGNs) in 
different channels are independently identically distributed (i.i.d) with the variance $\sigma^{2}$. The received SNR resulting from the direct transmission from the source to the destination in the first time slot is $\gamma_{s, d}[1]=p_{s, 1}[1] g_{s d} / \sigma^{2}$.And the received cooperative SNR of the source at the destination helped by the $i$ th relay in the second time slot is then $\Gamma_{s(i)}=\gamma_{s, d}[1]+\gamma_{s, i, d}$, where $\gamma_{s, i, d}=\frac{p_{s, 1}[1] g_{s i} p_{i, 1}[2] g_{i d}}{\sigma^{2}\left(p_{s, 1}[1] g_{s i}+p_{i, 1}[2] g_{i d}+\sigma^{2}\right)}$,is the relayed SNR.

At the same time, the relay also transmits its own information. We assume the relay establishes this communication using BD2. The received SNR resulting from the direct transmission at the AP in the first slot and the second slot can be expressed as $\gamma_{i, d}[1]=p_{i, 2}[1] g_{i d} / \sigma^{2}$, and $\gamma_{i, d}[2]=p_{i, 2}[2] g_{i d} / \sigma^{2}$. Without loss of generality, we use unit channel bandwidth to facilitate our discussion, and then the transmission rates of the source and the relay can be expressed as $R_{s}\left(p_{s, 1}[1], p_{i, 1}[2]\right)=\frac{1}{2} \log _{2}\left(1+\Gamma_{s(i)}\right)$, and $R_{i}\left(p_{i, 2}[1], p_{i, 2}[2]\right)=\frac{1}{2}\left[\log _{2}\left(1+\gamma_{i, d}[1]\right)+\log _{2}\left(1+\gamma_{i, d}[2]\right)\right] \cdot$ respectively.

The network utility using the $i$ th relay can be expressed as $T_{i}\left(p_{s, 1}[1], p_{i, 2}[1], p_{i, 1}[2], p_{i, 2}[2]\right)=R_{s}+R_{i}$, and the transmission rates of the source and the relay should satisfy

$$
\begin{aligned}
& R_{s} \geq R_{s, \text { min }}, \\
& R_{i} \geq R_{i, \text { min }},
\end{aligned}
$$

where $R_{s, \min }$ and $R_{i, \min }$ are the minimum transmission rate requirements of the source and the relay, respectively. To protect the PU, the transmit power of the source and the relay on each band must satisfy

$$
p_{s, 1}[1] g_{s p}, p_{i, 2}[1] g_{i p}, p_{i, 1}[2] g_{i p}, p_{i, 2}[2] g_{i p} \leq I,
$$

where $I$ is the acceptable received interference power of the PU, $g_{s p}$ and $g_{i p}$ are the channel coefficients from the source to the PU, and the relay to the PU, respectively.

Furthermore, due to battery capacity and radio front-end (RF) capability, there are total transmit power constraints for the source and the relay

$$
\begin{aligned}
& p_{s, 1}[1] \leq p_{s}^{\text {total }}, \\
& p_{i, 2}[1] \leq p_{i}^{\text {total }}, \\
& p_{i, 1}[2]+p_{i, 2}[2] \leq p_{i}^{\text {total }},
\end{aligned}
$$

where $p_{s}^{\text {total }}$ and $p_{i}^{\text {total }}$ are the total transmit power limits for the source and the relay, respectively.

\section{Transmit Power Constraints and System Throughput for Scenario II}

In Scenario II the full duplex can be realized because the two hops, work in orthogonal spectrum bands and the relay is able to transmit and receive simultaneously. As shown in Fig.1, we assume there are four different bands, BD1, BD2, BD3 and BD4. We assume that BD2 and BD3 are used as a dual-hop channel, and the source and the relay send their own data to the destination through BD1 and BD4 directly.

Denote $p_{s, j}$ and $p_{i, j}$ as the transmit power of the source and the relay for $\mathrm{BD} j, j=1,2,3,4$. Then their power allocation vectors can be expressed as $\mathbf{p}_{\mathbf{s}}=\left[p_{s, 1}, p_{s, 2}, p_{s, 3}, p_{s, 4}\right]$, and $\mathbf{p}_{\mathbf{i}}=\left[p_{i, 1}, p_{i, 2}, p_{i, 3}, p_{i, 4}\right]$, where $p_{s, 3}=p_{s, 4}=p_{i, 1}=p_{i, 2}=0$. Similarly, the received SNRs at the destination from the dual-hop transmission, the direct transmission from the source to the destination, and the direct transmission from the relay to the destination can be expressed as $\gamma_{s, i, d}=\frac{p_{s, 2} g_{s i} p_{i, 3} g_{i d}}{\sigma^{2}\left(p_{s, 2} g_{s i}+p_{i, 3} g_{i d}+\sigma^{2}\right)}, \gamma_{s, d}=p_{s, 1} g_{s d} / \sigma^{2}$, $\gamma_{i, d}=p_{i, 4} g_{i d} / \sigma^{2}$. Therefore, the transmission rates of the source and the relay can be expressed as $R_{s}\left(p_{s, 1}, p_{s, 2}, p_{i, 3}\right)=\log _{2}\left(1+\gamma_{s, i, d}\right)+\log _{2}\left(1+\gamma_{s, d}\right), R_{i}\left(p_{i, 4}\right)=\log _{2}\left(1+\gamma_{i, d}\right)$. Then the system throughput using the $i$ th relay can be denoted as $T_{i}\left(p_{s, 1}, p_{s, 2}, p_{i, 3}, p_{i, 4}\right)=R_{s}+R_{i}$. The constraints can be expressed as follows

$$
R_{s} \geq R_{s, \min },
$$




$$
\begin{aligned}
& R_{i} \geq R_{i, \text { min }}, \\
& p_{s, 1}+p_{s, 2} \leq p_{s}^{\text {total }}, \\
& p_{i, 3}+p_{i, 4} \leq p_{i}^{\text {total }}, \\
& p_{s, 1} g_{s p}, p_{s, 2} g_{i p}, p_{i, 3} g_{i p}, p_{i, 4} g_{i p} \leq I .
\end{aligned}
$$

\section{Relay Selection and Power Allocation}

\section{Optimization Problem Formulation}

Our objective is to maximize the system throughput by selecting the most profitable relay and designing corresponding suitable values of power allocation vectors $\mathbf{p}_{\mathbf{s}}$ and $\mathbf{p}_{\mathbf{i}}$. According to the previous discussion, this joint optimization problem can be formulated as $i^{*}=\underset{i \in\{1,2, \ldots, L\}}{\operatorname{argmax}} \operatorname{pax}_{\mathbf{i}} \in \Omega\left(T_{i}\right.$, where $\Omega$ is the feasible region defined by constraints Eq. 1-6 for Scenario I and constraints Eq. 7-11 for Scenario II. This is a joint optimization over the relay indexes and the transmit powers. However, we can perform this optimization in two steps: first over the transmit powers and then over the relay indexes. Accordingly, we first address the maximization of system throughput with respect to the transmit powers for a given relay candidate.

\section{Power Allocation}

\section{Optimal Power Allocation for Scenario I}

Noticing that $T_{i}$ is a monotonically increasing function of $p_{s, 1}[1]$ and $p_{i, 2}[1]$ and according to Eq. 3-5, we have $p_{s, 1}^{*}[1]=\min \left(I / g_{s p}, p_{s}^{\text {total }}\right)$ and $p_{i, 2}^{*}[1]=\min \left(I / g_{i p}, p_{i}^{\text {total }}\right)$. Then by substituting the optimal value $p_{s, 1}^{*}[1]$ and $p_{i, 2}^{*}[1]$ into $T_{i}$, the power allocation problem can be stated as

$$
\arg \max _{p_{i, 1}[2], p_{i, 2}[2]} T_{i}\left(p_{i, 1}[2], p_{i, 2}[2]\right) \text {, }
$$

Subject to

$$
\begin{aligned}
& p_{i, 1}[2] \leq I / g_{i p}, \\
& p_{i, 2}[2] \leq I / g_{i p}, \\
& p_{i, 1}[2]+p_{i, 2}[2] \leq p_{i}^{\text {total }}, \\
& R_{s} \geq R_{s, \text { min }}, \\
& R_{i} \geq R_{i, \text { min }} .
\end{aligned}
$$

Due to the concavity of logarithmic function, we know that $f_{1}(x)=-R_{i}\left(p_{i, 2}^{*}[1], x\right)$ is a convex function and we can also prove that $f_{2}(x)=-R_{s}\left(p_{s, 1}^{*}[1], x\right)$ is convex. Since $f_{1}(x)$ and $f_{2}(x)$ are convex functions, $-T_{i}$ and nonlinear inequality constraints Eq. $13 \mathrm{~d}$ and $13 \mathrm{e}$ are all convex. Eq. 12 is then a convex optimization problem, so the Karush-Kuhn-Tucker (KKT) conditions are both necessary and sufficient for this problem. If the feasible region determined by $p_{i, 1}[2] \geq 0, p_{i, 2}[2] \geq 0$ and constraints Eq. 13 is not empty, we apply the Lagrange multiplier method [8], and the Lagrange function for Eq. 12 is given by

$$
\begin{aligned}
& L\left(p_{i, 1}[2], p_{i, 2}[2], \lambda_{1}, \lambda_{2}, \lambda_{3}, \lambda_{4}, \lambda_{5}\right) \\
= & -T_{i}\left(p_{s, 1}^{*}[1], p_{i, 2}^{*}[1], p_{i, 1}[2], p_{i, 2}[2]\right)+\lambda_{1}\left(p_{i, 1}[2]-I / g_{i p}\right)+\lambda_{2}\left(p_{i, 2}[2]-I / g_{i p}\right) \\
& +\lambda_{3}\left(p_{i, 1}[2]+p_{i, 2}[2]-p_{i}^{\text {total }}\right)+\lambda_{4}\left(R_{s, \text { min }}-R_{s}\left(p_{s, 1}^{*}[1], p_{i, 1}[2]\right)\right)+\lambda_{5}\left(R_{i, \text { min }}-R_{i}\left(p_{i, 2}^{*}[1], p_{i, 2}[2]\right)\right),
\end{aligned}
$$


where $\lambda_{m}, m=1, . ., 5$ are the introduced Lagrange multipliers. By taking the partial derivative of $L$ with $p_{i, 1}[2], p_{i, 2}[2]$ and according to the KKT conditions, we obtain $\frac{\partial L}{\partial p_{i, j}[2]}=0, j=1,2$. Now, we define the following variables: $b_{1}=p_{s, 1}^{*}[1] g_{s i}+\sigma^{2}, b_{2}=p_{s, 1}^{*}[1] g_{s d}+\sigma^{2}$ and $b_{3}=\frac{p_{s, 1}^{*}[1] g_{s i} g_{i d}\left(p_{s, 1}^{*}[1] g_{s i}+\sigma^{2}\right)\left(1+\lambda_{4}\right)}{2\left(\lambda_{1}+\lambda_{3}\right) \ln 2}$.

Then direct calculation yields

$$
\begin{aligned}
& p_{i, 1}[2]=\left[-\frac{b_{1}\left(b_{1}+2 b_{2}-\sigma^{2}\right)}{2\left(b_{1}+b_{2}-\sigma^{2}\right) g_{i d}}+\frac{\sqrt{b_{1}^{2}\left(b_{1}-\sigma^{2}\right)^{2}+4 b_{3}\left(b_{1}+b_{2}-\sigma^{2}\right)}}{2\left(b_{1}+b_{2}-\sigma^{2}\right) g_{i d}}\right]^{+}, \\
& p_{i, 2}[2]=\left[\frac{1+\lambda_{5}}{2\left(\lambda_{2}+\lambda_{3}\right) \ln 2}-\frac{\sigma^{2}}{g_{i d}}\right]^{+},
\end{aligned}
$$

where $x \in[0,+\infty)$.

Using the iterative algorithm, $p_{i, 1}[2], p_{i, 2}[2]$ can be updated through Eq. 15 while $\lambda_{m}$ can be obtain using the sub-gradient method [8] in each step given by

$$
\begin{aligned}
& \lambda_{1}^{(n+1)}=\left[\lambda_{1}^{(n)}+\alpha^{(n)}\left(p_{i, 1}^{(n)}[2]-I / g_{i p}\right)\right]^{+}, \\
& \lambda_{2}^{(n+1)}=\left[\lambda_{2}^{(n)}+\alpha^{(n)}\left(p_{i, 2}^{(n)}[2]-I / g_{i p}\right)\right]^{+}, \\
& \lambda_{3}^{(n+1)}=\left[\lambda_{3}^{(n)}+\alpha^{(n)}\left(p_{i, 1}^{(n)}[2]+p_{i, 2}^{(n)}[2]-p_{i}^{\text {total }}\right)\right]^{+}, \\
& \lambda_{4}^{(n+1)}=\left[\lambda_{4}^{(n)}+\alpha^{(n)}\left(R_{s, \text { min }}-R_{s}\left(p_{s, 1}^{*}[1], p_{i, 1}^{(n)}[2]\right)\right)\right]^{+}, \\
& \lambda_{5}^{(n+1)}=\left[\lambda_{5}^{(n)}+\alpha^{(n)}\left(R_{i, \text { min }}-R_{i}\left(p_{i, 2}^{*}[1], p_{i, 2}^{(n)}[2]\right)\right)\right]^{+},
\end{aligned}
$$

where $\alpha^{(n)}$ is a small positive step size for the $n$th iteration. With appropriate step sizes, the iterations are proved to be convergent [9]. Once convergent $\lambda_{m}$ are obtained, we can get the optimal power allocation $p_{i, 1}^{*}[2], p_{i, 2}^{*}[2]$ and the corresponding $T_{i}^{*}$ when the $i$ th relay is selected.

\section{Optimal Power Allocation for Scenario II}

In this scenario, the power allocations at the source and the relay interact with each other. In fact, the non-convexity of the constraint Eq. 7 and the objective function $T_{i}$ makes this power allocation problem non-convex, and hence hard to solve. In order to tackle this problem, we introduce a power proportionality factor $0 \leq \theta \leq 1$ at the relay to divide its total transmit power into two parts, $\theta p_{i}^{\text {total }}$ and $(1-\theta) p_{i}^{\text {total }}$, for the source information relaying and its own data transmission, respectively. By this means, this problem is decomposed into two levels. At low level, we can design an optimal source power allocation for a given relay power allocation which is fixed by $\theta$. Then, at high level, the system throughput can be maximized by searching for the optimal $\theta$.

Now we develop the optimal source power allocation algorithm for a given $\theta=\theta_{0}$ at low level. Considering the PU interference constraints Eq. 11, we obtain

$$
\begin{aligned}
& p_{i, 3}\left(\theta_{0}\right)=\min \left\{\theta_{0} p_{i}^{\text {total }}, I / g_{\text {ip }}\right\}, \\
& p_{i, 4}\left(\theta_{0}\right)=\min \left\{\left(1-\theta_{0}\right) p_{i}^{\text {total }}, I / g_{\text {ip }}\right\} .
\end{aligned}
$$

Then constraint Eq. 8 can be converted into the following linear inequality

$$
p_{i, 4}\left(\theta_{0}\right) \geq\left(2^{R_{i, \text { min }}}-1\right) \sigma^{2} / g_{i d} \text {. }
$$

If the value of $\theta_{0}$ meets (19), the source power allocation problem can be formulated as follows.

$$
\left(p_{s, 1}^{*}, p_{s, 2}^{*}\right)=\arg \max _{p_{s, 1}, p_{s, 2}} T_{i}\left(p_{s, 1}, p_{s, 2}, \theta\right) \text {, }
$$

Subject to

$$
\begin{aligned}
& p_{s, 1} \leq I / g_{s p}, \\
& p_{s, 2} \leq I / g_{s p},
\end{aligned}
$$




$$
\begin{aligned}
& p_{s, 1}+p_{s, 2} \leq p_{s}^{\text {total }}, \\
& R_{s}\left(p_{s, 1}, p_{s, 2}, p_{i, 3}\left(\theta_{0}\right)\right) \geq R_{s, \text { min }} .
\end{aligned}
$$

Similar to that in Scenario I, we can prove Eq. 20 is a convex problem. Using the Lagrange multiplier method, the Lagrange function for Eq. 20 is given by

$$
\begin{aligned}
& L\left(p_{s, 1}, p_{s, 2}, \mu_{1}, \mu_{2}, \mu_{3}, \mu_{4}\right) \\
& \quad=-T_{i}\left(p_{s, 1}, p_{s, 2}, \theta_{0}\right)+\mu_{1}\left(p_{s, 1}-I / g_{s p}\right)+\mu_{2}\left(p_{s, 2}-I / g_{s p}\right), \\
& \quad+\mu_{3}\left(p_{s, 1}+p_{s, 2}-p_{s}^{\text {total }}\right)+\mu_{4}\left(R_{s, \min }-R_{s}\left(p_{s, 1}, p_{s, 2}, p_{i, 3}\left(\theta_{0}\right)\right)\right.
\end{aligned}
$$

Applying the KKT conditions, then we can obtain the closed-form solution to (36) as

$$
\begin{aligned}
& p_{s, 1}=\left[\frac{1+\mu_{4}}{\left(\mu_{1}+\mu_{3}\right) \ln 2}-\frac{\sigma^{2}}{g_{i d}}\right]^{+}, \\
& p_{s, 2}=\frac{\sigma^{2}}{g_{s i}}\left[\frac{-c_{1}+\sqrt{c_{1}^{2}-4 c_{2}}}{2}\right]^{+},
\end{aligned}
$$

where

$$
c_{1}=2+p_{i, 3}\left(\theta_{0}\right) g_{i d} / \sigma^{2} \text {, and } c_{2}=1+p_{i, 3}\left(\theta_{0}\right) g_{i d}\left[\frac{1}{\sigma^{2}}-\frac{\left(1+\mu_{4}\right) g_{s i}}{\sigma^{4}\left(\mu_{2}+\mu_{4}\right) \ln 2}\right] \text {. }
$$

Using the sub-gradient method, we can further obtain convergent $\mu_{m}$ through iteration,

$$
\begin{aligned}
& \mu_{1}^{(n+1)}=\left[\mu_{1}^{(n)}+\alpha^{(n)}\left(p_{s, 1}^{(n)}-I / g_{s p}\right)\right]^{+}, \\
& \mu_{2}^{(n+1)}=\left[\mu_{2}^{(n)}+\alpha^{(n)}\left(p_{s, 2}^{(n)}-I / g_{s p}\right)\right]^{+}, \\
& \mu_{3}^{(n+1)}=\left[\mu_{3}^{(n)}+\alpha^{(n)}\left(p_{s, 1}^{(n)}+p_{s, 2}^{(n)}-p_{s}^{\text {total }}\right)\right]^{+}, \\
& \mu_{4}^{(n+1)}=\left[\mu_{4}^{(n)}+\alpha^{(n)}\left(R_{s, \min }-R_{s}\left(p_{s, 1}^{(n)}, p_{s, 2}^{(n)}, p_{i, 3}\left(\theta_{0}\right)\right)\right]^{+} .\right.
\end{aligned}
$$

Once convergent $\mu_{m}$ are obtained, we can get the optimal power allocation $p_{s, 1}^{*}\left(\theta_{0}\right), p_{s, 2}^{*}\left(\theta_{0}\right)$ at the source and corresponding system throughput $T_{i}\left(p_{s, 1}^{*}\left(\theta_{0}\right), p_{s, 2}^{*}\left(\theta_{0}\right), \theta_{0}\right)$.

At high level, our aim is to find the optimal value of the power proportionality factor $\theta^{*}$, and the problem can be modeled as

$$
\theta^{*}=\arg \max _{\theta \in \Psi} T_{i}(\theta),
$$

where $\Psi$ is the feasible region defined by $0 \leq \theta \leq 1$ and constraint Eq. 19 .

This problem can be solved by search method [10]. Since $T_{i}(\theta)$ is not unimodal, we adopt a modified golden section search algorithm in [11] to obtain $\theta^{*}$ in this paper. Then, by substituting $\theta^{*}$ and corresponding convergent $\mu_{m}$ into Eq. 17, 18, and 23, the optimal power allocation between the source and the relay can be obtained when the $i$ th relay is selected.

\section{Simulation Results}

In this section, simulation results are presented to demonstrate the effectiveness of the proposed algorithms.In particular, we compare the performance of our algorithms with that of random relay selection with optimal power allocation (OPA) and equal power allocation (EPA). The OPA is performed according to the description in Sect. 3.2. The EPA means that the total transmit power of each node is uniformly distributed among all its occupied bands.

\section{Simulation Parameters}

In the simulation, we consider the scenario in which $L$ relay candidates are randomly distributed between the source node and the AP in a rectangular region. The channel between each node introduces fading and propagation path loss. The fading is an i.i.d. Rayleigh random variable with a unit variance. The propagation path loss is defined as the power law of the distance between the 
transmitting node and the receiving node, and the path-loss exponent equals 2 . All the channel coefficients vary slowly in time. The back ground noise is an i.i.d complex Gaussian random variable and the noise level is $-110 \mathrm{dBm}$. We set the PU received interference power limit $I=-70 \mathrm{dBm}$.

\section{Simulation Results and Discussions}

Taking Scenario I for example, we first evaluate the performance of the three-node network in terms of system throughput.

Fig. 2 presents the performance of the proposed scheme in terms of system throughput with respect to the total transmit power limit for the relay, $p_{i}^{\text {total }}$. In our simulation, the number of relay candidates is $L=10$ and we set the total transmit power limit for the source is $p_{s}^{\text {total }}=10 \mathrm{dBm}$. As observed in this figure, the proposed scheme results in better system throughput compared to the other schemes. The optimal scheme achieves about $45 \%$ throughput gain over the random relay selection with EPA and about $30 \%$ throughput gain over the random relay selection with OPA. Moreover, the suboptimal scheme has only about $10 \%$ throughput degradation and has almost the same performance when $p_{i}^{\text {total }}$ is high. In high $p_{i}^{\text {total }}$ region, the system throughput increases with $p_{i}^{\text {total }}$ slowly. This is because the system throughput is restricted by $I$ and not sensitive to $p_{i}^{\text {total }}$. However, in low $p_{i}^{\text {total }}$ region, the system throughput increases rapidly with $p_{i}^{\text {total }}$.

Fig. 3 shows that the system throughput versus the total transmit power at the source, $p_{s}^{\text {total }}$. In the simulation, the total transmit power limit for each relay candidate is fixed at $p_{i}^{\text {total }}=10 \mathrm{dBm}$, and the number of relay candidates is $L=10$. From the figure, the system throughput increases with the total transmit power at the source. This is because the system throughput is mainly determined by the total transmit power at the source when the total transmit power at the relay is a constant. In high $p_{s}^{\text {total }}$ region, we can also see that the system throughput increases with $p_{s}^{\text {total }}$ slowly.

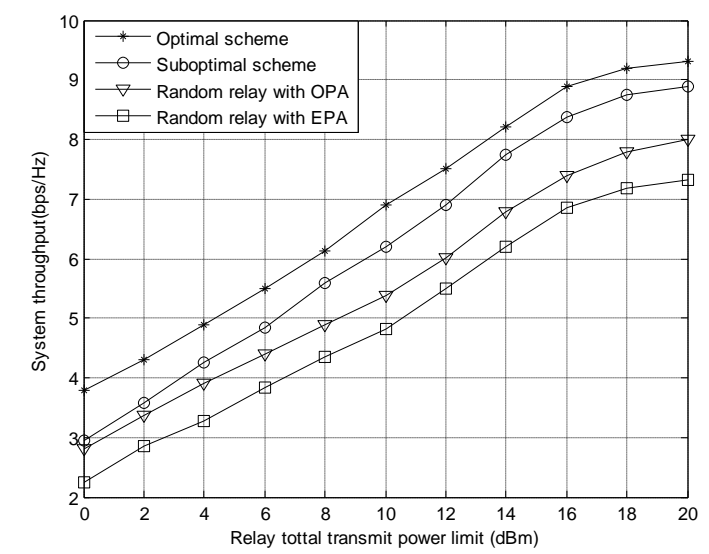

Fig. 2 System throughput versus relay total transmit power limit. 


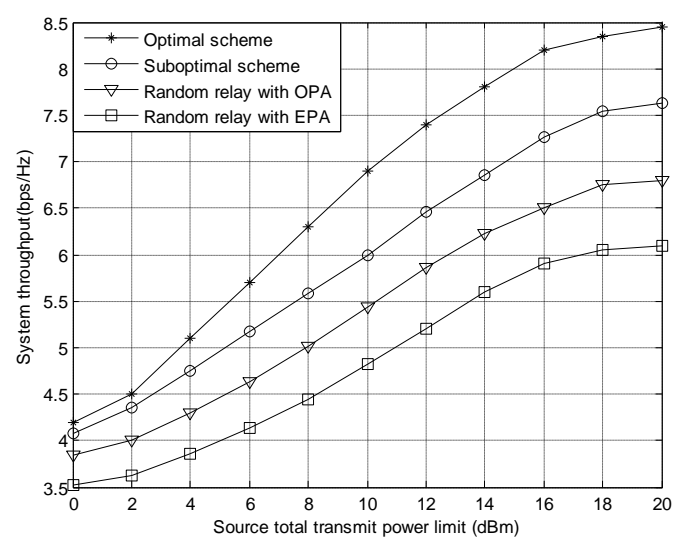

Fig. 3 System throughput versus source total transmit power limit.

\section{Conclusion}

In this paper, we have investigated the problem of joint relay selection and power allocation for CR systems with considering the performance of the SU acts as the relay. Two scenarios where the relay node involves relay diversity and dual-hop transmission have been considered. The optimal schemes have been obtained to achieve the maximum system throughput while supporting the QoS requirements of both the source and the relay. Accordingly, the close-form expressions for the optimal power allocation have been derived in both the scenarios. The simulation results presented in Sect. 4 adequately demonstrate the advantages of the proposed algorithms.

\section{References}

[1] A. Goldsmith, S. Jafar, I. Maric, and S. Srinivasa, "Breaking spectrum gridlock with cognitive radios: an information theoretic perspective," Proc. IEEE, vol. 97, pp. 894-914, May 2009.

[2] K. B. Letaief and W. Zhang, "Cooperative communications for cognitive radio networks," Proc. IEEE, vol. 97, no. 5, pp. 878-893, May 2009.

[3] Q. Zhang, J. Jia, and J. Zhang, "Cooperative relay to improve diversity in cognitive radio networks,” IEEE Commun. Mag., vol. 47, no. 2, pp. 111-117, Feb. 2009.

[4] K. J. Kim, T. Q. Duong and X. Tran, "Performance analysis of cognitive spectrum-sharing single-carrier systems with relay selection," IEEE Trans. Signal Process., vol. 60, no. 12, pp. 6435-6449, Dec. 2012.

[5] L. Li, X. Zhou, H. Xu, and G. Y. Li, "Simplified relay selection and power allocation in cooperative cognitive radio systems," IEEE Trans. Wireless Commun., vol. 10, no. 1, pp. 33-36, Jan. 2011.

[6] D. Bharadia, G. Bansal, P. Kaligineedi, and V. K. Bhargava, "Relay and power allocation schemes for OFDM-based cognitive radio systems," IEEE Trans. Wireless Commun., vol. 10, no. 9, pp. 2812-2817, Sep. 2011

[7] G. Zhao, C. Yang, G. Y. Li, D. Li, and A. C. K. Soong, "Power and channel allocation for cooperative relay in cognitive radio networks," IEEE J. Sel. Topics Signal Process. , vol. 5, no. 1, pp. 151-159, Feb. 2011.

[8] D. P. Palomar and M. Chiang, "A tutorial on decomposition methods for network utility maximization,” IEEE J. Sel. Areas Commun., vol. 24, no. 8, pp. 1439-1451, Aug. 2006.

[9] D. Bertsekas, Nonlinear Programming. Belmont, MA: Athena Scientific, 1999. 
[10] M. A. Bhatti, Practical Optimization Methods: With Mathematica Applications. Springer, 2000.

[11] E. Hopfinger, "On the solution of the unidimensional local minimization problem," Journal of Optimization Theory and Applications, vol. 18, no. 3, pp. 425-428, Nov. 1976. 